\title{
Camarões de água doce de bacias hidrográficas do cerrado: distribuição geográfica e chave de identificação
}

Os camarões de água doce do gênero Macrobrachium (Spence Bate, 1868) encontram-se amplamente distribuídos nas Américas e, numericamente este é o gênero dominante da família Palaemonidae. O Estado do Maranhão não dispõe de trabalhos voltados para crustáceos de água doce, sendo este o primeiro estudo realizado com o objetivo de apresentar informações taxonômicas das espécies registradas, com mapa de ocorrência geográfica, características biológicas e ecológicas, uma chave de identificação do gênero Macrobrachium para o Estado, bem como aspectos de conservação. Para isso, oito riachos do Leste do Maranhão, foram amostrados com puçás, peneiras e rede de arrasto, passados no fundo e na vegetação marginal submersa por um período de 20 minutos em um trecho de $150 \mathrm{~m}$. Em laboratório, os espécimes foram identificados, mensurados e sexados. Um total de 526 espécimes foram coletados, distribuídos em cinco espécies, sendo: M. carcinus ( $N=01)$, M. olfersii $(N=55), M$. acanthurus $(N=116), M$. amazonicum $(N=119)$ e M. jelskii $(N=235)$. Somente em $M$. acanthurus e $M$. jelskii foram registradas fêmeas ovígeras, M. jelskii apresentou fecundidades de 1 a 65 ovos, ao passo que para a única fêmea ovígera de M. acanthurus foram contados 662 ovos. Também foi elaborado uma chave de identificação do gênero Macrobrachium para o Maranhão. Assim, confirmou-se os registros destas espécies para o Estado e ampliação da área de distribuição, com mapas de ocorrência.

Palavras-chave: Diversidade; Decapoda; Macrobrachium; Taxonomia; Conservação.

\section{Freswater shrimps from the cerrado hydrographic basins: geographical distribution and key of identification}

\begin{abstract}
Freshwater shrimp for genus, Macrobrachium (Spence Bate, 1868) are widespread in the Americas and, numerically, this is the dominant genus of the Palaemonidae family. The State of Maranhão doesn't have research associated with freshwater crustaceans, with this being the first study period carried out with the objective of showing registered taxonomic species information and map of geographic occurrences, biological and ecological characteristics, a Macrobrachium genus identification key for the State, as well as conservation aspects. For this, eight streams in eastern Maranhão were sampled with gill nets, sifters and dragnets being ran across the bottom and in submersed marginal vegetation for a period of 20 minutes along a 150-meter stretch. In the laboratory, specimens were identified, measured and sexed. A total of 526 specimens were collected, distributed in five species, being: $M$. carcinus ( $N=01), M$. olfersii ( $N=55)$, M. acanthurus $(\mathrm{N}=116), \mathrm{M}$. amazonicum ( $\mathrm{N}=119$ ) and $\mathrm{M}$. jelskii $(\mathrm{N}=235)$. Ovigerous females were only registered in $\mathrm{M}$. acanthurus and $\mathrm{M}$. jelskii, $\mathrm{M}$. jelskii showed fertilities from 1 to 65 eggs, while for the only ovigerous female from M. acanthurus, 662 eggs were counted. An identification key of the genus Macrobrachium was also prepared for Maranhão. Thus, the records of these species for the State and expansion of the distribution area were confirmed, with occurrence maps.
\end{abstract}

Keywords: Diversity; Decapoda; Macrobrachium; Taxonomy; Conservation.

Topic: Conservação da Biodiversidade

Reviewed anonymously in the process of blind peer
Received: 02/05/2020

Approved: 02/06/2020
Kele Sousa Pires Andrade (iD

Universidade Federal do Maranhão, Brasil http://lattes.cnpq.br/7515596782122130

http://orcid.org/0000-0001-7083-3962

kelesousapires@gmail.com

Ivaneide de Oliveira Nascimento (iD)

Universidade Estadual da Região Tocantina do Maranhão, Brasil

http://lattes.cnpq.br/5127803057876571

http://orcid.org/0000-0001-7095-7092

ivaneide_agro@yahoo.com.br

Marina de Sá Leitão Câmara de Araújo (iD

Universidade de Pernambuco, Brasil

http://lattes.cnpq.br/4315354380176563

http://orcid.org/0000-0002-0966-4423

mslc.araujo@gmail.com
Jorge Luiz Silva Nunes (iD)

Universidade Federal do Maranhão, Brasi

http://lattes.cnpq.br/1899258151972278

http://orcid.org/0000-0001-6223-1785

silvanunes@yahoo.com
Referencing this:

ANDRADE, K. S. P.; NASCIMENTO, I. O.; ARAÚJO, M. S. L. C.; NUNES, J. L. S.. Camarões de água doce de bacias hidrográficas do cerrado: distribuição geográfica e chave de identificação. Revista Ibero Americana de Ciências Ambientais, v.11, n.4, p.102-113, 2020. DOI: http://doi.org/10.6008/CBPC2179-6858.2020.004.0009 


\section{INTRODUÇÃO}

O gênero Macrobrachium (Spence Bate, 1868) possui ampla distribuição nas Américas, compreende cerca de 240 espécies e, os mais diversos da família Palaemonidae (HOLTHUIS, 1952; MELO, 2003; VALENCIA et al., 2007; ANGER, 2013; SAENGPHAN et al., 2018). Ocorre em rios e córregos de interiores e águas salobras (MELO, 2003; PILEGGI et al., 2012). Este gênero possui espécies de importância econômica (VALENCIA et al., 2007; LIMA et al., 2013), distintos padrões fisiológicos de adaptação e características reprodutivas (MOSSOLIN et al., 2010; ANGER, 2013). Dentre as 23 espécies de Macrobrachium registradas para o Brasil, das quais duas são espécies introduzidas, três se destacam com potencial para exploração econômica por atingirem maior porte e, portanto maior rendimento de carne: Macrobrachium carcinus (Linnaeus, 1758), Macrobrachium acanthurus (Wiegmann, 1836) e o Macrobrachium amazonicum (Heller, 1862) (VALENCIA et al., 2007; MANTELATTO et al., 2008; BENTES et al., 2011; LOEBMANN et al., 2010; MACIEL et al., 2011; ANGER, 2013; SANTOS et al., 2013; ROSSI et al., 2020).

Os camarões do gênero Macrobrachium foram registrados no Maranhão desde o final do século XX, inicialmente por Ramos-Porto et al. (1978) ao estudarem os crustáceos pertencentes a Coleção Carcinológica do Laboratório de Hidrobiologia da Universidade Federal do Maranhão que registraram as espécies $M$. acanthurus e M. amazonicum. Nesse intervalo de quase 40 anos, os camarões de água doce têm sido objeto de pequena parte de estudos sobre crustáceos decápodes no Estado (COELHO et al., 1980; COELHO et al., 1985; MELO, 2003; SOUSA et al., 2013, SOUSA et al., 2015; ANDRADE et al., 2017) com registro de espécies do gênero Macrobrachium: M. acanthurus (Wiegmann, 1836), M. amazonicum (Heller, 1862), M. brasiliense (Heller, 1862) e M. jelskii (Miers, 1877). E apesar do Estado está inserido em uma transição biogeográfica (AB'SABER, 1989), tendo representação de três dos seis biomas brasileiros, o bioma Amazônia, Caatinga e Cerrado (IBGE, 2004) e, apresentar uma rede hidrográfica bem distribuída, o conhecimento sobre a comunidade de decápodes dulcícolas nessa região ainda se encontra incipiente (ANDRADE et al., 2017).

Logo, o objetivo desse estudo é contribuir com informações taxonômicas do gênero Macrobrachium registradas para o Estado, com o fornecimento de informações sobre a história natural (tamanho, distribuição, habitat e características reprodutivas) e uma chave de identificação para as espécies reportadas.

\section{MATERIAIS E MÉTODOS}

As coletas foram realizadas entre Outubro de 2014 a Março de 2015, em cinco riachos no município de Chapadinha (Riacho Itamacaoca, Riacho da Prata, Riacho Feio, Riacho Canto Escuro e Riacho Repouso), um riacho no município de Paulino Neves (Riacho São José), um riacho no município de Tutóia (Riacho Água Rica) e um riacho no município de Barreirinhas (Riacho Passagem do Canto), Estado do Maranhão, todos em região do Cerrado (Licença de № 42415-1 MMA/ICMBIO). Os pontos de amostragem localizados nos municípios de Barreirinhas, Tutóia e Paulino Neves estão inseridos na Bacia do Rio Preguiças. E os pontos de coleta do município de Chapadinha estão inseridos na Bacia do Rio Munim (NUGEO, 2011) (Fig. 1). 


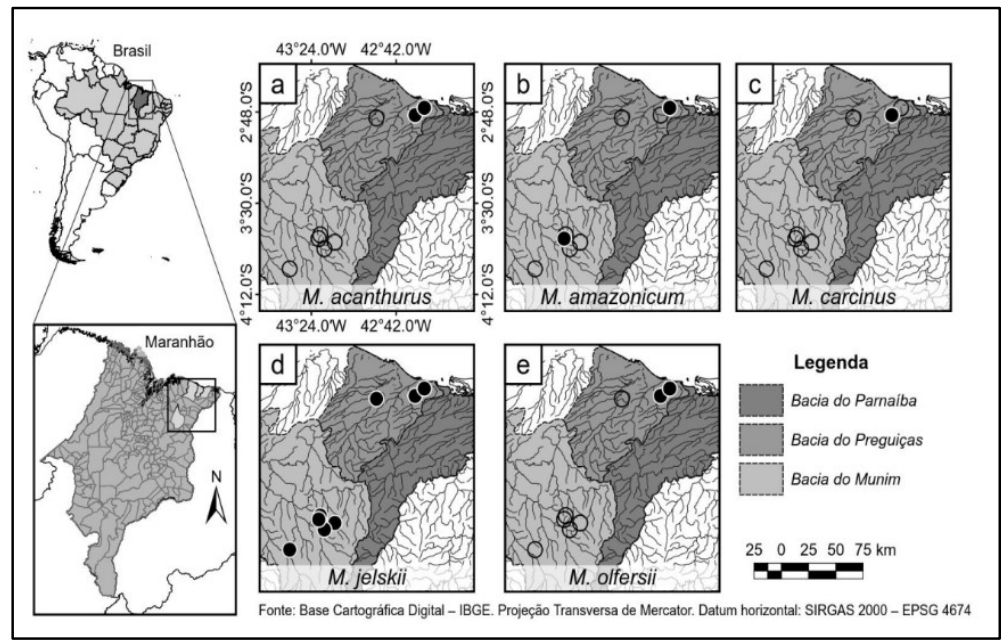

Figura 1: a - e. Mapa de ocorrência de camarões Macrobrachium, Estado do Maranhão. a) Ocorrência do $M$. acanthurus (círculos pretos), b) Ocorrência do M. amazonicum (círculos pretos), c) Ocorrência do M. carcinus (círculo preto), d) Ocorrência do M. jes/kii (círculos pretos), e) Ocorrência do M. olfersii (círculos pretos). Os Círculos Vazios simbolizam os pontos amostrais do estudo e os Círculos Pretos simbolizam as ocorrências das espécies.

Os usos mais comuns dos recursos hídricos são para o abastecimento urbano e turismo. A expansão do turismo trouxe para a região empreendimentos imobiliários, residências e pousadas, que estão sendo construídos na orla do rio principal. Essas construções, bem como a abertura de trilhas por carros de tração são utilizadas para transportar turistas e têm contribuído para retirada da vegetação ripária (SANTOS et al., 2013).

Cada riacho foi coletado uma única vez, com uso de puçás de malha de $1 \mathrm{~cm}$, peneiras com $52 \mathrm{~cm}$ de diâmetro e malha de $0,4 \mathrm{~cm}$ e também se utilizou uma rede de arrasto com abertura de malha de $0,17 \mathrm{~cm}$, os quais foram passados sob a vegetação marginal e na água por um período de 20 minutos em um trecho de $150 \mathrm{~m}$ determinados de cada riacho.

Durante as coletas foram mensurados os parâmetros físicos e químicos, potencial hidrogeniônico $(\mathrm{pH})$, oxigênio dissolvido e temperatura $\left({ }^{\circ} \mathrm{C}\right)$, por meio do medidor multiparâmetro. Os espécimes coletados foram separados por ponto amostral, etiquetados e fixados em solução de formol de $10 \%$ e posteriormente conservados em álcool 70\%. Os espécimes foram identificados segundo Holthuis (1952), García-Dávila et al. (2003) Melo (2003) e Sampaio et al. (2009). O sexo de cada espécime foi verificado com base na presença (machos) ou ausência (fêmeas) do apêndice masculino localizado no segundo par de pleópodos. Foram separados em machos, fêmeas não-ovígeras e fêmeas ovígeras.

Após a determinação do sexo de cada indivíduo, foram aferidas as medidas do comprimento do cefalotórax (CCF) - extremo distal do rostro ao extremo posterior do cefalotórax, comprimento total (CT) medida entre as extremidades distais do rostro e da extremidade do telson) com um paquímetro digital $(0,01 \mathrm{~mm})$ e peso total $(\mathrm{PT})$ em balança digital com precisão $0,01 \mathrm{~g}$.

Espécimes foram depositados na Coleção Didática de Zoologia (CDZ) da Universidade de Pernambuco, Campus Garanhuns (CDZUPE611, CDZUPE 612, CDZUPE 613, CDZUPE 614 e CDZUPE 615). As medidas que constam entre parênteses no item 'Material coletado e examinado' se referem às medidas do cefalotórax. 


\section{RESULTADOS}

Das 23 espécies de camarões do gênero Macrobrachium reportadas para o Brasil, cinco ocorreram no presente estudo, num total de 526 espécimes: M. acanthurus (Wiegmann, 1836), Macrobrachium amazonicum (Heller, 1862), M. carcinus (Linnaeus, 1758), M. jelskii (Miers, 1877) e M. olfersii (Wiegmann, 1836) (Fig. 1). Desse modo, segue abaixo a chave de distribuição das espécies de Macrobrachium para o Maranhão.

Tabela 1: Chave de identificação para machos adultos do gênero Macrobrachium do Leste do Maranhão, Brasil:

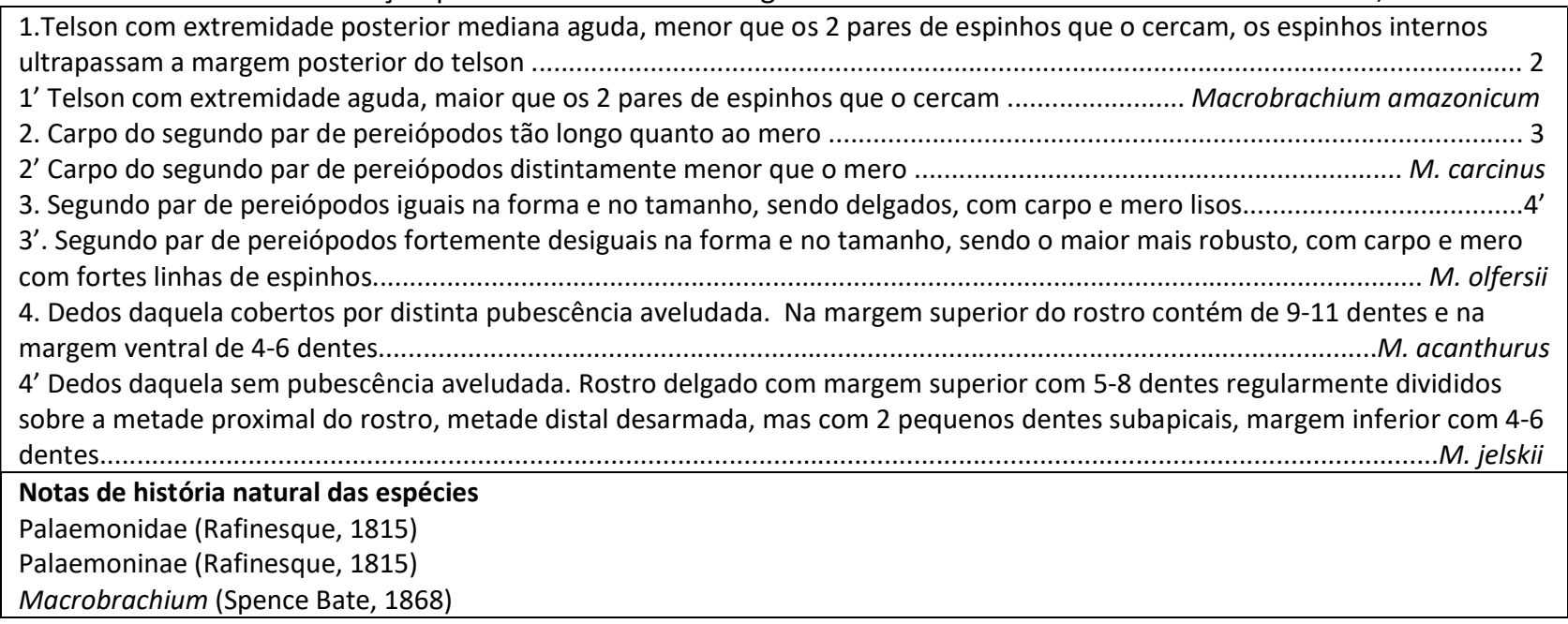

\section{O Macrobrachium acanthurus (Wiegmann, 1836)}

Material coletado e examinado: Brasil, Maranhão, Tutóia: Riacho Água Rica (S 02 ${ }^{\circ} 46^{\prime} 04.3^{\prime \prime} \mathrm{O}$ 042 28'04,0"), 19.X.2014, 40 ơ (10,95 a 40,47 mm), 73 ९ (6,33 a 34,66 mm) 1 ๆ ovígera (33,65 mm), Brasil, Maranhão, Paulino Neves: Riacho São José (0249'26.2"S 042³2'38.3"O), 20.X.2014, 2 ơ $^{\circ}$ (12,32 e 30,18 mm). Comprimento dos espécimes: Machos 10,95 a 40,47mm (CCF) e de 25,96 a 86,88mm (CT), Fêmeas 6,33 a 34,66mm (CCF) e de 15,70 a 75,59mm (CT), Fêmea ovígera 33,65mm (CCF) e 68,89mm (CT). Das 74 fêmeas registradas, somente 01 era ovígera com tamanho corporal $68,69 \mathrm{~mm}$, portanto 662 ovos.

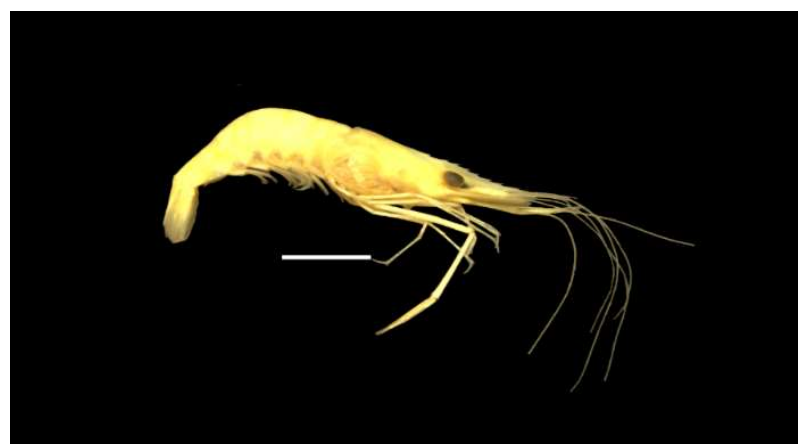

Figura 2: Macrobrachium acanthurus (Wiegmann, 1836), Macho, CDZUPE 614, Riacho Água Rica - Tutóia (escala $0,9 \mathrm{~mm})$.

\section{Diagnose: (MELO, 2003)}

Habitat: Os espécimes foram registrados em ambientes que apresentaram gramíneas, pequenos arbustos, serrapilheira nas margens dos riachos. Tendo como substrato composto por areia, matéria 
orgânica, pequenas raízes e lodoso, propiciando abrigos pedaços de madeiras, raízes, banco de folhas e plantas aquáticas. Estes ambientes apresentaram água com oxigênio dissolvido 4,48 a 4,95 mg/l, pH 8,58 a 8,69 e temperaturas 23,23 a $25,39 \circ \mathrm{C}$.

\section{O Macrobachium amazonicum (Heller, 1862)}

Material coletado e examinado: Brasil, Maranhão, Tutóia: Riacho Água Rica (S 02 46'04.3" O 042 $\left.28^{\prime} 04,0^{\prime \prime}\right), 19 . X .2014,39 \sigma^{\top}(16,07$ a 27,94 mm) 75 ᄋ (13,03 a 45,08 mm), Brasil, Maranhão, Chapadinha: Riacho do Repouso (346'15.62"S 43²0'23.71"O, 24.III.2015, 4 ơ (14,95 a 20,65mm) 1 ㅇ (19,33mm). Comprimento dos espécimes: Machos: 14,95 a 27,94mm (CCF) e de 31,03 a 58,89mm (CT), Fêmeas: 13,03 a 45,08mm (CCF) e de 29,66 a 74,05mm (CT).

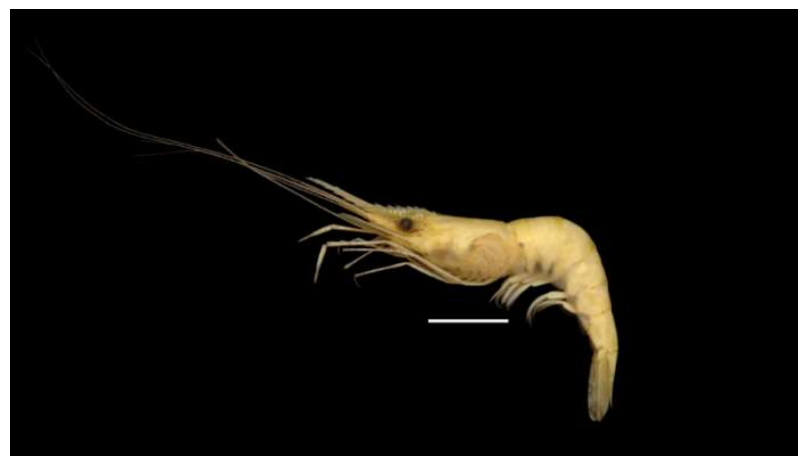

Figura 3: Macrobachium amazonicum (Heller, 1862), Fêmea, CDZUPE 612, Riacho Água Rica - Tutóia (escala 1,3mm).

\section{Diagnose (MELO, 2003)}

Habitat: Os espécimes foram encontrados em riachos com pequenos arbustos, arvores e gramínea nas margens. Tendo como substrato areia, matéria orgânica, raízes finas, macrófitas e abrigos para os camarões como pedaços de madeiras, raízes e plantas aquáticas. A água destes ambientes é transparente com oxigênio dissolvido 3,17 a 4,48, pH 8,54 a 8,69 e temperaturas entre 23,23 a 26,8으.

\section{Macrobrachium carcinus (Linnaeus, 1758)}

Material coletado e examinado: Brasil, Maranhão, Paulino Neves: Riacho São José (0249'26.2"S 042³2'38.3"O), 20.X.2014, 1 ơ (67,36mm). Comprimento dos espécimes: Macho: 67,36 mm (CCF) e $169,15 \mathrm{~mm}(\mathrm{CT})$.

Diagnose: (MELO, 2003)

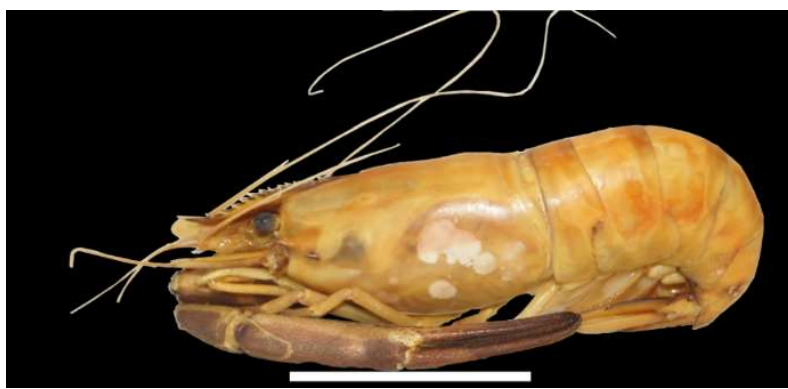

Figura 4: Macrobrachium carcinus (Linnaeus, 1758), Macho, CDZUPE 615, Riacho São José - Paulino Neves (escala $36,5 \mathrm{~mm})$ 
Hábitat: No presente estudo, o espécime foi encontrado no riacho São José, o qual apresenta gramíneas, pequena quantidade de árvores e pequenos arbustos em suas margens. Tendo o substrato lodoso, com matéria orgânica e raízes. Possui abrigos para os camarões como pedaços de madeiras e bancos de folhas. A água deste riacho apresentou oxigênio dissolvido 4,95 mg/l, pH 8,58 e temperatura 25,39으.

\section{O Macrobrachium olfersii (Wiegmann, 1836)}

Material coletado e examinado: Brasil, Maranhão, Tutóia: Riacho Água Rica (S 02 46'04.3" O 042 28'04,0"), 19.X.2014, 13 \% (5,81 a 16,74mm), 18 ơ (9,87 a 24,31mm), Brasil, Maranhão, Paulino Neves: Riacho São José $\left(02^{\circ} 49^{\prime} 26.2^{\prime \prime S} 042^{\circ} 32^{\prime} 38.3^{\prime \prime O}\right), 20 . X .2014,21 o^{\pi}$ (9,35 a 23,62 mm), 3 9 (7,26 a 13,18 mm). Comprimento dos espécimes: Machos: 9,35 a 24,31mm (CCF) e de 17,96 a 60,18 mm (CT), Fêmeas: 5,81 a $16,74 \mathrm{~mm}$ (CCF) e de 15,12 a $41,93 \mathrm{~mm}(\mathrm{CT})$.

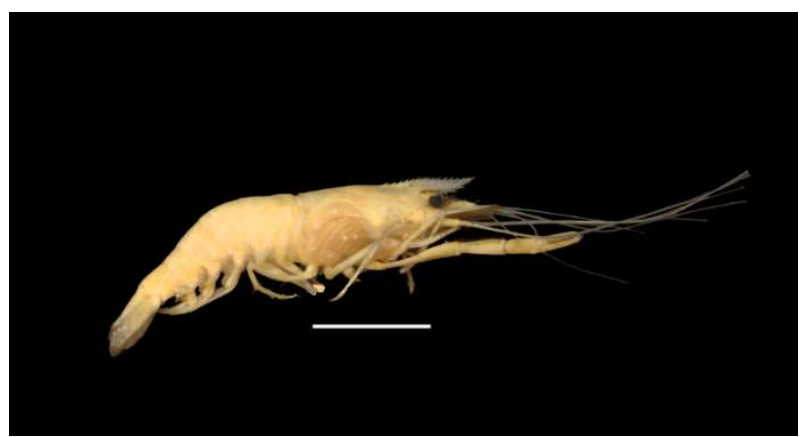

Figura 5: Macrobrachium olfersii (Wiegmann, 1836), Macho, CDZUPE 613, Riacho São José - Paulino Neves (escala $1,4 \mathrm{~mm})$.

\section{Diagnose: (MELO, 2003; VALENCIA et al., 2007)}

Hábitat: Neste estudo, os espécimes foram encontrados em ambientes que apresentam pequenos arbustos, gramíneas, serrapilheira nas margens dos riachos. Tendo o substrato arenoso, matéria orgânica, pequenas raízes e lodoso, propiciando abrigos pedaços de madeiras, raízes, banco de folhas e as plantas aquáticas para os camarões. Estes ambientes apresentaram água com oxigênio dissolvido 4,48 a 4,95, pH 8,58 a 8,69 e temperaturas 23,23 a 25,39 ㅇ․

\section{O Macrobrachium jelskii (Miers, 1877)}

Material coletado e examinado: Brasil, Maranhão, Tutóia: Riacho Água Rica (S 02 ${ }^{\circ} 46^{\prime} 04.3^{\prime \prime} \mathrm{O}$ 042²8'04,0"), 19.X.2014, 4 ९ (7,42 a 14,37mm), 1 ovígera (15,01mm), Brasil, Maranhão, Chapadinha:

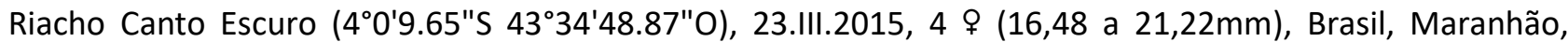
Paulino Neves: Riacho São José (0249'26.2"S 042³2'38.3"O), 20.X.2014, 37 \$ (7,14 a 16,44mm) , 30 ơ (7,46 a 14,76mm), 22 @ ovígeras (13,01 a 16,42mm) , Brasil, Maranhão, Barreirinhas: Riacho Passagem do Canto (02 $\left.{ }^{\circ} 50^{\prime} 45.2^{\prime \prime S} 042^{\circ} 51^{\prime} 48.8^{\prime \prime O}\right), 22 . X .2014,22$ \% (10,66 a 15,75mm), 5 \% ovígeras (13,86 a 15,69mm), 17 o

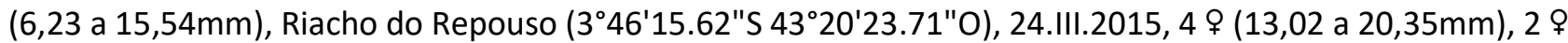

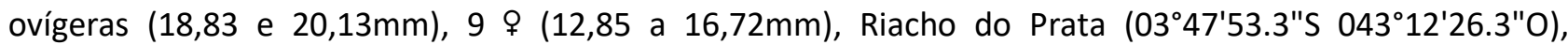


21.VIII.2014, 16 (9,04 a 19,21mm), 23 ovígeras (8,91 a 19,38mm), 7 ơ (9,14 a 15,63mm), Riacho

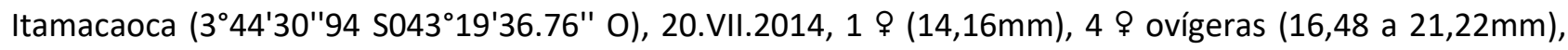

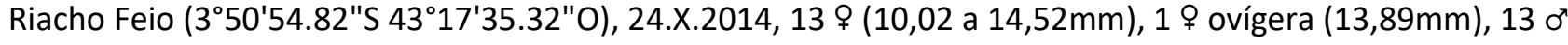
$(8,42$ a $17,06 \mathrm{~mm})$.

Comprimento dos espécimes: Machos: 6,23 a 17,06 mm (CCF) e de 17,86 a 42,08 mm (CT), Fêmeas: 6,23 a 20,35 mm (CCF) e de 16,42 a 48,66mm (CT), fêmeas ovígeras 8,91 a 21,22 mm (CCF) e de 14,67 a 48,37mm (CT). Foram capturadas 58 fêmeas ovígeras, com comprimento corporal de 14,67 a 48,37 mm, com fecundidade entre 1 a 65 ovos.

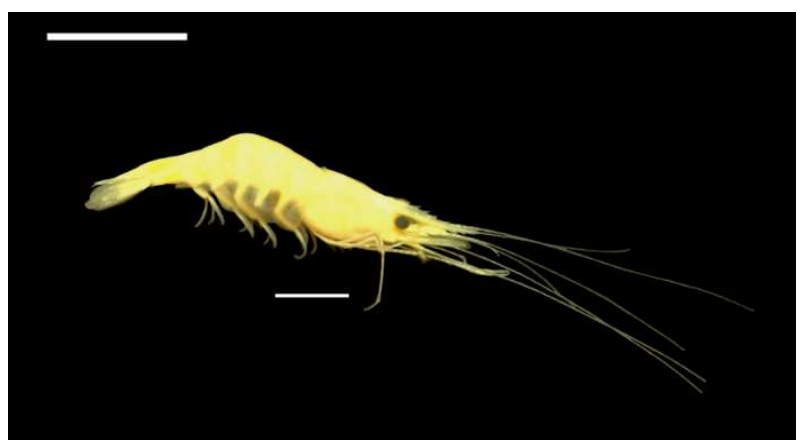

Figura 6: Macrobrachium jelskii (Miers, 1877), Fêmea, CDZUPE 611, Riacho Canto Escuro - Chapadinha (escala de $0,8 \mathrm{~mm})$.

\section{Diagnose: (MELO 2003)}

Hábitat: Foi a única espécie registrada nos oito riachos, confirmando que é uma espécie generalista, segundo Coelho et al. (1985). No presente estudo, os espécimes foram encontrados em ambientes em que a água apresentou o oxigênio dissolvido 1,79 a 9,92, $\mathrm{pH} 3,85$ a 8,88 e temperaturas 23,23 a 27,34으.

Variações morfológicas: Um total de 42 espécimes apresentaram 4 dentes na parte inferior do rostro, sendo 19 espécimes no riacho São José, 8 no Riacho Passagem do Canto e 3 espécimes no riacho Água Rica, riachos pertencentes a Bacia do Rio Preguiças. Já na Bacia do Rio Munin, 8 espécimes no riacho Feio e 4 no riacho Repouso apresentaram a característica de possuir 4 dentes na parte inferior do rostro. Em espécimes maiores, o rostro apresentou variação da porção distal, estando ligeiramente inclinado para cima, alcançando ou ultrapassando o escafocerito, já em espécimes menores, esta situação é irregular.

\section{DISCUSSÃO}

O gênero Macrobrachium se distribui de forma distinta, no Indo-Pacífico, África Ocidental e nas Américas, tendo como principal fator limitante de distribuição a temperatura da água (MULLER et al., 1992; ANGER, 2013) o que claramente coincide com as correntes oceânicas quentes que atuam no Atlântico, como a Corrente do Golfo, e a Corrente Norte do Brasil, ou no norte Equatorial contracorrente no Pacífico (ANGER, 2013). A preferência das espécies do gênero por águas quentes é bem documentada na literatura e também encontra base nos dados ambientais do material coletado neste estudo, tendo temperaturas entre 16,7 a 37으 (COELHO et al., 1985; MOSSOLIN et al., 2002; MEJíA-ORTíz et al., 2010; BENTES et al., 2011; BARROSALVES et al., 2012; NÓBREGA et al., 2013; NERY et al., 2015; SOARES et al., 2015). 
As cinco espécies coletadas neste estudo, já haviam sido registradas para o estado do Maranhão (MELO, 2003; PILEGGI et al., 2013; ANDRADE et al., 2017), sendo que as espécies $M$. acanthurus, $M$. amazonicum tinham sido registradas somente na costa do Estado e em ambientes marinho-estuarino. Um único exemplar de $M$. carcinus foi registrado no riacho São José em Paulino Neves, acredita-se que esse número mínimo de espécime coletado se deve a dificuldade em coletá-lo com redes e puçás, geralmente coleta-se com tipo de armadilhas tipo covo (ROCHA, 2010).

Dentre as espécies registradas, somente o $M$. jelskii possui o desenvolvimento larval abreviado, apresenta menor número de ovos, com maiores volumes e dimensões, representando uma menor taxa de fecundidade (COELHO et al., 1985; MAGALHÃES, 2000; MONTOYA, 2003; MOSSOLIN et al., 2013; NERY et al., 2015). Já as demais espécies, $M$. acanthurus, M. amazonicum, M. carcinus e $M$. olfersii compartilham o mesmo comportamento no ciclo reprodutivo, sendo considerados diádromos (SHORT, 2004; BAUER, 2011), com desenvolvimento prolongado, portando ovos pequenos e numerosos nos pleópodos (MELO, 2003). Estas espécies são típicas de bacias hidrográficas que drenam para o Oceano Atlântico (HOLTHUIS, 1952; BOWLES et al., 2000; MELO, 2003). Por outro lado, somente o M. olfersii possui distribuição anfi-ístmica, com ocorrência na costa Atlântica quanto na Pacífica (ANGER, 2013; GARCÍA-VELAZCO et al., 2014).

Todas as quatro espécies diádromas registradas no presente estudo foram coletadas em duas localidades, especificamente nos municípios de Tutóia e Paulino Neves na Bacia do Rio Preguiças, e sua presença nessas localidades pode ser explicado pela intensa influência marinha (MOSSOLIN et al., 2002; MELO, 2003). Nestes dois pontos amostrais, houve o compartilhamento de todas as cinco espécies registradas neste estudo, como registrado por Almeida et al. (2008).

O compartilhamento de espécies entre esses pontos era um resultado esperado, uma vez que os pontos de coleta além de estarem a pouca distância (menos de $15 \mathrm{~km}$ ) um do outro, compartilham também aspectos de geomorfologia. Isso permite que padrões biogeográficos já bastante validados expliquem que a fauna aquática pode ter se mesclado nas várias drenagens próximas, o que inclui o baixo curso do rio Parnaíba, devido ao aumento de nível do mar, como as incursões marinhas do mioceno (HUBERT et al., 2006). Ainda segundo os autores, essa maior riqueza na Bacia do Rio Preguiças também pode ser explicada pelo gradiente de altitude, pois em áreas mais baixas tendem a possuir maior riqueza.

Neste estudo, a espécie $M$. amazonicum foi registrada em duas bacias hidrográficas em localidades com características limnológicas bem distintas, com pontos amostrais no curso médio da bacia do Rio Munim (cerca de 150 km da linha de costa) e no município de Tutóia, já sob influência marinha (15km em linha reta até a linha de costa). Esta ocorrência pode ser explicada, devido à espécie $M$. amazonicum ser dividida em duas populações, uma costeira e outra continental (MACIEL et al., 2009), por possuir ampla distribuição, grande variabilidade morfológica e amplitude de comportamento (COELHO et al., 1985)

As larvas planctônicas marinhas das espécies diádromas destacam-se pela alta dispersão através do fluxo de riachos e rios costeiros (BAUER, 2011), assim a conectividade entre ambientes de água doce e marinha proporciona as condições favoráveis ao ciclo reprodutivo dessas espécies e, portanto, as perturbações/degradação sofridas por esses habitats pode afetar a manutenção das espécies. 
Por outro lado, são também consideradas vulneráveis pela exigência da migração jusante a montante, o que acrescenta o risco de severas alterações na dinâmica reprodutiva serem causadas por meio da interferência antrópica como construção de barragens, desvios e outras formas de controle de riachos (BOWLES et al., 2000; MARCH et al., 2003; BAUER, 2011).

Dentre os camarões palaemonídeos, somente para o Macrobrachium carcinus (Linnaeus, 1758) são conhecidas ações de conservação (MANTELATTO et al., 2016). O M. carcinus consta no Livro Vermelho da Fauna Brasileira Ameaçada de Extinção desde o ano de 2012, incluso na categoria de Dados Insuficientes (DD), a qual é definida para definir o grau de extinção das espécies (ICMBıO, 2018). De acordo com a Instrução Normativa $n^{\circ}$ 5, de 21 de maio de 2004 do Ministério do Meio Ambiente, o Macrobrachium carcinus esta espécie segue ameaçada de extinção, para os estados de Pernambuco, Rio de Janeiro, Santa Catarina, Espírito Santo, Bahia, Alagoas, Pará, Piauí, Rio Grande do Sul, São Paulo, Ceará e Sergipe.

\section{CONCLUSÕES}

O presente estudo é o primeiro com o foco totalmente voltado para conhecimento da fauna de crustáceos de água doce no Leste do estado do Maranhão. Neste estudo foram registradas cinco espécies de camarões do gênero Macrobrachium: M. acanthurus, M. amazonicum, M. carcinus, M. jelskii e M. olfersii. Assim fornecendo uma extensão da distribuição destes. Além do fornecimento de uma chave de identificação e mapa de distribuição para o gênero Macrobrachium no Estado. Somente o M. brasiliense (Heller, 1862), com ocorrência para o Estado, não foi registrado no presente trabalho.

No decorrer do estudo, verificou-se a inexistência de trabalhos voltados para o conhecimento da biologia reprodutiva de camarões de água doce para o Estado. Ademais, os poucos trabalhos disponíveis se tratam de registros pontuais e esparsos, o que enfatiza a importância da execução de inventários da fauna de crustáceos para o Estado. Também é necessário realizar estudos sobre os camarões de interiores para auxiliar na compreensão de aspectos populacionais e biológicos, como exemplo do M. jelskii e de outras espécies tipicamente dulcícolas, são importantes para comparação de mudanças no meio que degradem os habitats.

Investir na aquicultura é outra medida, pois contribui para conservar as populações de camarões palaemonídeos, bem como para a conservação dos ecossistemas. No entanto, o que dificulta a produção dessas espécies em escala comercial é conseguir larvas, e por serem zooplanctófagas, necessitam de alimento vivo, sendo difíceis em manter no cativeiro (GARCÍA-GUERRERO et al., 2013). Para resolver isto, investir em pesquisas de biologia reprodutiva dessas espécies (MOSSOLIN et al., 2002) e desenvolver projetos em conjunto com a comunidade no entorno de riachos, e ajudá-los a compreender a importância de cuidar desse recurso, do qual retiram o sustento, são fundamentais para a manutenção do equilíbrio do ambiente (GARCÍA-GUERRERO et al., 2013).

AGRADECIMENTOS: À Fundação de Amparo à Pesquisa e ao Desenvolvimento Científico e Tecnológico do Maranhão - FAPEMA (APP Universal № Processo 00754/13), pelo apoio financeiro a pesquisa de campo. À 
Universidade Federal do Maranhão, ao Laboratório de Organismos Aquáticos (LabAqua) e ao Laboratório de Ecologia e Sistemática de Peixes (LABESP) pelo suporte e infraestrutura, ao PPGBC pelas diversas oportunidades oferecidas. E ao ICMBio e MMA (SISBIO 42415-1) para a concessão da permissão para coleta dos camarões.

\section{REFERÊNCIAS}

AB'SABER A.. Zoneamento ecológico e econômico da Amazônia: questões de escala e método. Estudos Avançados, São Paulo, v.3, n.5, p.4-20, 1989. DOI: https://doi.org/10.1590/S0103-40141989000100002

ALMEIDA, A. O.; COELHO, P.A.; LUZ, J. R.; DOS SANTOS, J. T. A.; FERRAZ, N. R.. Decapod crustaceans in fresh waters of southeastern Bahia, Brazil. Revista de Biologia Tropical, San José, v.56, n.3, p.1225-1254, 2008.

ANDRADE, K. S. P.; ARAÚJO, M. S. L.C.; NUNES, J. L. S.. New records of Macrobrachium (Spence Bate, 1868) (Decapoda, Palaemonidae) from the northern coast of Brazil. Check List, Sofia, v.13, n.4, p.379-390, 2017. DOI:

https://doi.org/10.15560/13.4.379

ANGER, K.. Neotropical Macrobrachium (Caridea: Palaemonidae): on the biology, origin, and radiation of freshwater-invading shrimp. Journal of Crustacean Biology, v.33, n.2, p.151-183, 2013. DOI: https://doi.org/10.1163/1937240X-00002124

BARROS-ALVES, S. P.; ALMEIDA, A. C.; FRANSOZO, V.. Population biology of shrimp Macrobrachium jelskii (Miers, 1778) (Decapoda, Palaemonoidea) at the Grande River at northwest of the state of Minas Gerais, Brazil. Acta Limnologica Brasiliensia, Rio Claro, v.24, n.3, p.266-275, 2012. DOI: https://doi.org/10.1590/S2179$\underline{\text { 975X2012005000044 }}$

BAUER, R. T.. Amphidromy and migrations of freshwater shrimps. II. delivery of hatching larvae to the sea, return juvenile upstream migration, and human impact. New Frontiers in Crustacean Biology, Leiden, p.157-168, 2011. DOI: https://doi.org/10.1163/ej.9789004174252.i-354.115

BENTES, B. S.; MARTINELLI, J.M.; SOUZA, L. S.; CAVALCANTE, D. V.; ALMEIDA, M. C.; ISAAC, V. J.. Spatial distribution of the Amazon River prawn Macrobrachium amazonicum (Heller 1862) (Decapoda, Caridea, Palaemonidae) in two perennial creeks of an estuary on the northern coast of Brazil (Guajará Bay, Belém, Pará). Brazilian Journal of Biology, São Carlos, v.71, p.925-935, 2011. DOI: https://doi.org/10.1590/S1519$\underline{69842011000500013}$

BOWLES, D. E.; AZIZ, K.; KNIGHT, C. L.. Macrobrachium (Decapoda: Caridea: Palaemonidae) in the contiguous United States: a review of the species and an assessment of threats to their survival. Journal of Crustacean Biology, v.20, n.1, p.158-171, 2000. DOI: https://doi.org/10.1163/2002197599990025

COELHO, P. A.; RAMOS-PORTO, M.. Camarões de água doce do Brasil: distribuição geográfica. Revista Brasileira de Zoologia, Curitiba, v.2, v.6, p.405-410, 1985. DOI: https://doi.org/10.1590/S0101-81751984000200014
COELHO, P. A.; RAMOS-PORTO, M.. Crustáceos decápodos da costa do Maranhão, Brasil. Boletim do Instituto Oceanográfico, São Paulo, v.29, n.2, p.135-138, 1980. DOI: https://doi.org/10.1590/S0373-55241980000200028

GARCÍA-GUERRERO, M. U.; BECERRIL-MORALES, F.; VEGAVILLASANTE, F.; ESPINOSA-CHAURAND, L. D.. Los langostinos del género Macrobrachium con importancia económica y pesquera en América Latina: conocimiento actual, rol ecológico y conservación. Latin American Journal of Aquatic Research, Valparaíso, v.41, n.4, p.651-675, 2013. DOI: http://dx.doi.org/103856/vol41-issue4-fulltext-3

GARCÍA-DÁVILA, C. R.; MAGALHÃES, C.. Revisão taxonômica dos camarões de água doce (Crustacea: Decapoda: Palaemonidae, Sergestidae) da Amazônia Peruana. Acta Amazonica, Manaus, v.33, n.4, p.663-686, 2003. DOI: https://doi.org/10.1590/S0044-59672003000400013

GARCÍA-VELAZCO, H.; MAEDA-MARTÍNEZ, A. M.; OBREGÓNBARBOZA, H.; RODRÍGUEZ-ALMARAZ, G.; VILLALOBOSHIRIART, J. L.; MURUGAN, G.. Evidence of oceanic dispersal of a disjunctly distributed amphidromous shrimp in Western North America: first record of Macrobrachium occidentale from the Baja California Peninsula. Journal of Crustacean Biology, v.34, n.2, p.199-215, 2014. DOI: https://doi.org/10.1163/1937240X-00002217

HOLTHUIS, L. B.. A general revision of the Palaemonidae (Crustacea Decapoda Natantia) of the Americas. II. The subfamily Palaemoninae. Los Angeles: Occasional Papers of the Allan Hancock Foundation, 1952.

HUBERT, N.; RENNO, J-F.. Historical biogeography of South American freshwater fishes. Journal of Biogeography, v. 33, n. 8, p. 1414-1436, 2006. DOI: https://doi.org/10.1111/j.1365-2699.2006.01518.x

ICMBio. Instituto Chico Mendes de Conservação da Biodiversidade. Livro Vermelho da Fauna Brasileira Ameaçada de Extinção. Brasília: ICMBio, 2018.

LIMA, A. V. B.; GUERRA, A. G.; ALMEIDA, E. A.; TADDEI, F. G.; CASTIGLIONI, L.. Characterization of esterase patterns in hepatopancreas of three species of Macrobrachium (Palaemonidae). Biochemical Systematics and Ecology, v.47, p.132-138, 2013. Dol: https://doi.org/10.1016/i.bse.2012.10.006

LOEBMANN, D.; MAI, A. C. G.; LEE, J. T.. The invasion of five alien species in the Delta do Parnaiba Environmental Protection Area, Northeastern Brazil. Revista de Biologia Tropical, San José, v.58, n.3, p.909-923, 2010.

MACIEL, C. R.; VALENTI, W. C.. Biology, Fisheries, and Aquaculture of the Amazon River Prawn Macrobrachium amazonicum: A Review. Nauplius, Botucatu, v.17, v.2, p.62- 
79, 2009.

MACIEL, C. R.; QUADROS, M. L.; ABRUNHOSA, F.; BASTOS, S.; SHNEIDER, H.; SAMPAIO, I.. Occurrence of the Indo-Pacific freshwater prawn Macrobrachium equidens Dana 1852 (Decapoda, Palaemonidae) on the coast of Brazilian Amazonia, with notes on its reproductive biology. Anais da Academia Brasileira de Ciências, Rio de Janeiro, v.83, n.2, p.533-544, 2011. DOI: https://doi.org/10.1590/S0001$\underline{37652011000200013}$

MAGALHÃES, C.. Abbreviated larval development of Macrobrachium jelskii (Miers, 1877) (Crustacea: Decapoda: Palaemonidae) from the Rio Solimões floodplain, Brazil, reared in the laboratory. Nauplius, Botucatu, v.8, v.1, p.1-15, 2000.

MANTELATTO, F. L.; PILEGGI, L. G.; SUÁREZ, H.; MAGALHÃES, C.. First record and extension of the known distribution of the inland prawn, Macrobrachium aracamuni Rodríguez, 1982 (Decapoda, Palaemonidae) in Brazil. Crustaceana, Leiden, v.81, n.2, p.241-246, 2008. DOI: https://doi.org/10.1163/156854008783476206

MANTELATTO, F. L.; PILEGGI, L. G.; MAGALHÃES, C.; CARVALHO, F. L.; ROCHA, S. S.; MOSSOLIN, E. C.; ROSSI, N.; BUENO, S. L. S.. Avaliação dos Camarões Palemonídeos (Decapoda: Palaemonidae). In: PINHEIRO, M. A. A.; BOOS, H.. Livro Vermelho dos Crustáceos do Brasil: Avaliação 2010-2014. Porto Alegre: SBC, 2016. p.252-267.

MARCH, J. G.; BENSTEAD, J. P.; PRINGLE, C. M.; SCATENA, F. N.. Damming tropical island streams: problems, solutions, alternatives. Bioscience, v.53, n.11, p.1069-1078, 2003. DOI: https://doi.org/10.1641/00063568(2003)053[1069:DTISPS]2.0.CO;2

MEJIA-ORTÍZ, L. M.; ALVAREZ, F.. Seasonal patterns in the distribution of three species of freshwater shrimp, Macrobrachium spp., along an altitudinal river gradient. Crustaceana, Leiden, v.83, n.4, p.385-397, 2010. DOI: https://doi.org/10.1163/001121610X489368

MELO, G. A. S.. Manual de identificação dos crustacea decapoda de água doce do Brasil. São Paulo: Loyola, 2003.

MONTOYA, J. V.. Freshwater shrimps of the genus Macrobrachium associated with roots of Eichhornia crassipes (Water Hyacinth) in the Orinoco Delta (Venezuela). Caribbean Journal of Science, v.39, n.1, p.155-159, 2003.

MOSSOLIN, E. C.; BUENO, S. L. S.. Reproductive biology of Macrobrachium olfersi (Decapoda, Palaemonidae) in São Sebastião, Brazil. Journal of Crustacean Biology, v.22, n.2, p.367-376, 2002. DOI: https://doi.org/10.1163/20021975$\underline{99990244}$

MOSSOLIN, E. C.; PEIRÓ, D. F.; ROSSINGNOLI, M. O.; RAJAB, L. P.; MANTELATTO, F. L.. Population and reproductive features of the freshwater shrimp Macrobrachium jelskii (Miers, 1877) from São Paulo State, Brazil. Acta Scientiarum. Biological Sciences, v.35, n.3, p.429-436, 2013. DOI: https://doi.org/10.4025/actascibiolsci.v35i3.15815

MOSSOLIN, E. C.; PILEGGI, L. G.; MANTELATTO, F. L. Crustacea, Decapoda, Palaemonidae, Macrobrachium Bate, 1868, São Sebastião Island, state of São Paulo, southeastern
Brazil. Check List, v.6, p.605-613, 2010. DOI: http://dx.doi.org/10.15560/6.4.605

MULLER, Y. M. R.; PRAZERES, A. C.. Influência da salinidade e temperatura da água sobre a captura de Macrobrachium olfersi (Wiegmann, 1836) coletados no canal da Lagoa do Peri, Florianópolis/SC. Acta Limnologica Brasiliensia, Rio Claro, v.4, p.175-183, 1992.

NERY, M. F. G.; PINHEIRO, A. P.; SILVA, D. S.; DUARTE, A. R. R.; LUCENA, I. C.. Fecundity of the shrimp Macrobrachium jelskii (Miers, 1877) in the southern portion of the state of Ceará, Brazil. Scientia Plena, v.11, n.1, p.1-7, 2015.

NÓBREGA, P. S. V.; BENTES, B.; MARTINELLI-LEMOS, J. M.. Composition of shrimp populations (Crustacea: Decapoda) in non-vegetated areas of two river islands in a Brazilian Amazon estuary. Zoologia, Curitiba, v.30, n.6, p.645-651, 2013. DOI: https://doi.org/10.1590/S198446702013005000004

NUGEO. Núcleo Geoambiental. Bacias hidrográficas: Subsídios para o Planejamento e a Gestão Territorial. São Luís: NUGEO, 2011.

PILEGGI, L. G.; MANTELATTO, F. L.. Taxonomic revision of doubtful Brazilian freshwater shrimp species of genus Macrobrachium (Decapoda, Palaemonidae). Iheringia, Porto Alegre, v.102, n.4, p.426-437, 2012. DOI: https://doi.org/10.1590/S0073-47212012005000012

PILEGGI, L. G.; MAGALHÃES, C.; BOND-BUCKUP, G.; MANTELATTO, F. L.. New records and extension of the known distribution of some freshwater shrimps in Brazil. Revista Mexicana de Biodiversidad, Ciudad de México, v.84, n.2, p.563-574, 2013. DOI: https://doi.org/10.7550/rmb.30504

NÓBREGA, P. S. V.; BENTES, B.; MARTINELLI-LEMOS, J. M.. Composition of shrimp populations (Crustacea: Decapoda) in non-vegetated areas of two river islands in a Brazilian Amazon estuary. Zoologia, Curitiba, v.30, n.6, p.652-660, 2013. DOI: https://doi.org/10.1590/S198446702013005000004

RAMOS-PORTO, M.; CORREIA, M. M. F.; SOUSA, N. R.. Levantamento da fauna aquática da Ilha de São Luis (Estado do Maranhão, Brasil). Il Crustacea. Boletim do Laboratório de Hidrobiologia, São Luís, v. 1, n. 1, p. 77-89, 1978.

ROCHA, S. S.. Diferença entre dois métodos de coleta utilizados na captura de crustáceos decápodes em um rio da Estação Ecológica Juréia-Itatins, São Paulo. Iheringia, Série Zoologia, Porto Alegre, v. 100, n. 2, p. 116-122, 2010. DOI: https://doi.org/10.1590/S0073-47212010000200005

ROSSI, N.; MAGALHÃES, C.; MESQUITA, E. R.; MANTELATTO, F. L.. Uncovering a hidden diversity: a new species of freshwater shrimp Macrobrachium (Decapoda: Caridea: Palaemonidae) from Neotropical region (Brazil) revealed by morphological review and mitochondrial genes analyses. Zootaxa, v. 4732, n. 1, p. 177-195, 2020. DOI: https://doi.org/10.11646/zootaxa.4732.1.9

SAENGPHAN, N.; PANIJPAN, B.; SENAPIN, S.; LAOSINCHAI, P.; RUENWONGSA, P.; SUKSOMNIT, A.; PHIWSAIYA, K.. Morphology and molecular phylogeny of Macrobrachium 
suphanense sp. nov. (Decapoda: Palaemonidae) from Thailand. Zootaxa, n.4482, n.1, p.151-163, 2018.

DOI: http://dx.doi.org/10.11646/zootaxa.4482.1.7

SAMPAIO, S. R.; NAGATA, J. K.; LOPES, O. L.; MASUNARI, S.. Camarões de águas continentais (Crustacea, caridea) da Bacia do Atlântico oriental paranaense, com chave de identificação tabular. Acta Biológica Paranaense, Curitiba, v.38, n.1-2, p.11-34, 2009.

DOI: http://dx.doi.org/10.5380/abpr.v38i0.15974

SANTOS, L. C. A.; LEAL, A. C.. Gerenciamento de recursos hídricos no estado do Maranhão-Brasil. Observatorium: Revista Eletrônica de Geografia, Uberlândia, v.5, n.13, p.3965, 2013.

SANTOS, A.; HAYD, L.; ANGER, K.. A new species of Macrobrachium (Spence Bate, 1868) (Decapoda, Palaemonidae), M. pantanalense, from the Pantanal, Brazil. Zootaxa, Nova Zelândia, v.3700, n.4, p.534-546, 2013. DOI: http://dx.doi.org/10.11646/zootaxa.3700.4.2

SHORT, J. W.. A revision of Australian river prawns, Macrobrachium (Crustacea: Decapoda: Palaemonidae). Hydrobiologia, v.525, p.1-100, 2004. DOI: https://doi.org/10.1023/B:HYDR.0000038871.50730.95
SOARES, M. S.; OSHIRO, L. M. Y.; TOLEDO, J. C.. Biologia reprodutiva de Macrobrachium jelskii (Crustacea, Decapoda, Palaemonidae) no Rio São Francisco, Minas Gerais, Brasil. Iheringia, Série Zoologia, Porto Alegre, v.105, n.3, p.307315, 2015. DOI: https://doi.org/10.1590/1678476620151053307315

SOUSA, A. R.; ALMEIDA, Z. S.; SANTOS, N. B.; NUNES, J. L. S.. Crustáceos. In: NUNES, J. L. S.; MENDONÇA, M. A.. Biodiversidade marinha da Ilha do Maranhão. São Luís: EDUFMA, 2013. p.95-124.

SOUSA, D. B.; SANTOS, N. B.; OLIVEIRA, V. M.; CARVALHONETA, R. N. F.; ALMEIDA, Z. S.. Carcinofauna bêntica estuarina de dois manguezais da Costa Amazônica Maranhense, Brasil. Iheringia, Série Zoologia, Porto Alegre, v.105, v.3, p.339-347, 2015. DOI: https://doi.org/10.1590/1678-476620151053339347

VALENCIA, D. M.; CAMPOS, M. R.. Freshwater prawns of the genus Macrobrachium Bate, 1868 (Crustacea: Decapoda: Palaemonidae) of Colombia. Zootaxa, Nova Zelândia, v.1456, p.1-44, 2007.

DOI: http://dx.doi.org/10.11646/zootaxa.1456.1.1

A CBPC - Companhia Brasileira de Produção Científica (CNPJ: 11.221.422/0001-03) detém os direitos materiais desta publicação. Os direitos referem-se à publicação do trabalho em qualquer parte do mundo, incluindo os direitos às renovações, expansões e disseminações da contribuição, bem como outros direitos subsidiários. Todos os trabalhos publicados eletronicamente poderão posteriormente ser publicados em coletâneas impressas sob coordenação da Sustenere Publishing, da Companhia Brasileira de Produção Científica e seus parceiros autorizados. Os (as) autores (as) preservam os direitos autorais, mas não têm permissão para a publicação da contribuição em outro meio, impresso ou digital, em português ou em tradução. 